\title{
Review
}

\section{The Use of Mobile Applications as Communication Aids for People with Dementia: Opportunities and Limitations}

\author{
Anjay Ambegaonkar ${ }^{\mathrm{a}, *}$, Craig Ritchie $^{\mathrm{b}}$ and Sofia de la Fuente Garcia ${ }^{\mathrm{c}}$ \\ a Independent Researcher, Johns Hopkins University, Baltimore, MD, USA \\ ${ }^{\mathrm{b}}$ Centre for Clinical Brain Sciences, University of Edinburgh, Edinburgh, UK \\ ${ }^{\mathrm{c}}$ Usher Institute, University of Edinburgh, Edinburgh, UK
}

Accepted 5 July 2021

Pre-press 30 July 2021

\begin{abstract}
.
Background: Communication difficulties are one of the primary symptoms associated with dementia, and mobile applications have shown promise as tools for facilitating communication in patients with dementia (PwD). The literature regarding mobile health (mHealth) applications, especially communications-based mHealth applications, is limited.

Objective: This review aims to compile the existing literature on communications-based mobile applications regarding dementia and assess their opportunities and limitations. A PICO framework was applied with a Population consisting of PwD, Interventions consisting of communication technology, focusing primarily on mobile applications, Comparisons between patient well-being with and without technological intervention, and Outcomes that vary but can include usability of technology, quality of communication, and user acceptance.

Methods: Searches of PubMed, IEEE XPLORE, and ACM Digital Library databases were conducted to establish a comprehensive understanding of the current literature on dementia care as related to 1) mobile applications, 2) communication technology, and 3) communications-based mobile applications. Applying certain inclusion and exclusion criteria, yielded a set of articles $(n=11)$.

Results: The literature suggests that mobile applications as tools for facilitating communication in PwD are promising. Mobile applications are not only feasible socially, logistically, and financially, but also produce meaningful communication improvements in PwD and their caregivers. However, the number of satisfactory communications-based mobile applications in the mHealth marketplace and their usability is still insufficient.

Conclusion: Despite favorable outcomes, more research involving PwD using these applications are imperative to shed further light on their communication needs and on the role of mHealth.
\end{abstract}

Keywords: Caregiver, communication, dementia, mobile applications

\section{INTRODUCTION}

${ }^{*}$ Correspondence to: Anjay Ambegaonkar, 9 Molly Pitcher Rd, Marlboro, NJ 07746, USA. Tel.: +1 908 216 6331; E-mail: anjay_ 03@yahoo.com.
In recent years, dementia diagnosis has increased quite significantly and is projected to increase in the future [1]. The current lack of preventative medication or disease-modifying intervention places most of the burden on caregivers, and the literature suggests that as patients with dementia (PwD) cognitively 
deteriorate, caregivers face higher burden resulting in lower quality of life for both patients and themselves $[2,3]$. As current pharmacological treatment methods still prove to be inadequate in both preventing neurodegeneration onset and significantly slowing the progression of the disease, non-pharmacological support and interventions have emerged that attempt to improve cognitive function and general well-being in patients, thereby decreasing the burden on caregivers. Among these support methods is the facilitation of communication in PwD. Mobile applications are increasingly becoming the user interface by which such support methods can be administered.

Dementia is a category of neurodegenerative diseases known for causing memory loss, communication difficulties, and general cognitive decline. Communication difficulties are particularly severe in vascular dementia, which accounts for $17 \%$ of persons diagnosed with dementia [4]. Furthermore, in Alzheimer's disease (AD), the most frequent etiology of dementia (60-70\% cases), alterations of speech, language, and overall communication are also very prominent [5]. This loss of communication is mostly verbal, while non-verbal communication seems to be preserved in PwD [6]. Despite this, non-verbal communication is not always sufficient to express the needs of the patient. Thus, Information and Communication Technologies (ICT) have been examined thoroughly by the literature as potential tools for the creation of methods that could facilitate and restore the loss in communication between the patient and the caregiver. Lindberg et al. (2013) systematically reviewed the literature discussing ICT in home care and found that "[m]ost studies show that communication between healthcare professionals and patients living at home was improved by using various ICT applications, as improvement in management of symptoms in daily life. It was revealed that various ICT applications can be advantageous to use in follow-up care of patients at home." It was also noted that ICT applications were not a replacement for face-to-face communication, but merely a tool to facilitate it [7]. Similarly, among a variety of user interfaces including telephone-based, video-based, and computer based, various studies have shown that ICT intervention decreased depression, anxiety, and caregiver burden [8]. It is of note to delineate that telephone-based, video-based, and computer-based interventions are different from the mobile phone user interface and applications.

The types of technologies used to assist function in PwD include those aforementioned (telephone, video, and computer) but are also extended to GPS and tracking devices, cameras, and sensor devices [9]. Smartphones in particular are a promising technology used in care arrangements for PwD, as they include modern technological progress and demonstrate great versatility for PwD support. For example, Cunningham et al. (2019) designed a study on a mobile application called MemoryTracks that combines music therapy with the smartphone userinterface. The user-interface itself was designed for the caregiver and aimed to minimize the added burden to the caregiver as a result of using the app. Accordingly, the app was designed to be as clear and self-explanatory as possible. The study concluded that MemoryTracks was successful in eliciting more positive emotions in patients, and it even resulted in some patients being able to perform actions that had previously deteriorated, such as feeding oneself [10]. Additionally, Serra-Añó et al. (2019) showed a more technically demanding task fulfilled by a mobile phone. In this study, sensors on an Android device were sufficient in tracking the postural control and gait of patients with $\mathrm{AD}$ and showed that postural control and gait were worse in patients with $\mathrm{AD}$ as compared to the control group [11]. Despite the range of tasks that smartphone applications can fulfill regarding dementia care support, it must be acknowledged that drawbacks include the lack of technology savviness in the patient population, lack of sufficient access to these devices, and general lack of knowledge of the benefits of mobile applications in treating dementia [12]. Some of these drawbacks can be addressed with more research and more exposure of mobile applications to the patient population.

In sum, even partial restoration of communication among PwD has the potential to increase quality of life in PwD and their caregivers. Given that mobile technology has promise to enhance and facilitate communication in these vulnerable groups, this article will review the opportunities and limitations associated with communications-based mobile applications for dementia care and support. In addition, despite the growing mobile health application market, mHealth applications designed to aid communication in PwD are relatively sparse. Hence, this paper aims to review the existing literature and highlight the relevant avenues for future research.

\section{LITERATURE SEARCH METHODOLOGY}

The literature was relatively saturated with articles about 1) smartphone apps as support options 
for PwD and 2) communication technology for PwD $(n=5,002)$. However, articles about communication and mobile applications together in aiding PwD were less common $(n=1,556)$. Originally, the literature search was selective only to communications-based mobile applications in the literature, but given the lack of publications, it was expanded to include articles about the efficacy of mobile applications as support options and articles about other communication technologies as support options, respectively. Still, only a handful of articles met the determined criteria.

Two separate literature searches were performed each in the PubMed, IEEE XPLORE, and ACM Digital Library databases, respectively. Search I utilized the compound Boolean search query (1) ("Dementia" AND "Mobile Applications") OR (2) ("Dementia" AND "Communication Technology"). Search II consisted of another Boolean search query, namely "Dementia" AND "Mobile Applications" AND "Communication Technology". The following criteria were used to screen the records resulting from these searches:

\section{Search I: ("Dementia" AND "Mobile}

Applications") OR ("Dementia" AND

"Communication Technology”)

\section{Inclusion criteria}

- Discusses PwD or caregiver interaction with mobile application or communication technology;

- Discusses efficacy/availability of mobile applications or communication technology in PwD;

- Contains information related to mobile applications or communication technology as it relates to dementia care;

- Is a review paper that offers insight into the logistics of mobile applications or communication technologies for dementia care.

\section{Exclusion criteria}

- Discusses mobile applications and communication technology that do not relate to PwD or caregiver interaction;

- Discusses mobile applications and communication technology specifically geared towards caregiver and not PwD;

- Discusses mobile applications and communication technology solely related to dementia diagnosis.
Search II: "Dementia” AND "Communication

Technology" AND "Mobile Applications"

\section{Inclusion criteria}

- Describes and analyzes specific case studies of communications-based mobile applications being used to assist conversation in PwD;

- Discusses feasibility of communications-based mobile applications in PwD;

- Describes and analyzes specific case studies of communication-based mobile applications being used to assist necessity-based communication in PwD.

\section{Exclusion criteria}

- Relates solely to design process of communications-based mobile application(s);

- Does not contain information about a single mobile application related to communication or useful information that could be deduced about communications-based mobile applications for dementia care.

For Search I in the PubMed database, the first Boolean search query ("Dementia" AND "Mobile Applications") yielded 110 articles, and the second Boolean search query ("Dementia" AND "Communication Technology") yielded 1,400 articles, for a total of 1,510 articles. Both queries were subjected to the same screening method, outlined by the inclusion and exclusion criteria for Search I, and six articles were selected for review.

Search II yielded a total of 32 articles and following a second screening method, outlined by the inclusion and exclusion criteria for Search II, three articles were selected for review. The two searches were then aggregated for a total of nine eligible articles.

The process was repeated for the IEEE XPLORE and ACM Digital Library. In Search I of the IEEE XPLORE database, the first Boolean search query ("Dementia" AND "Mobile Applications") yielded 68 articles, and the second Boolean search query ("Dementia" AND "Communication Technology") yielded 214 articles, for a total of 282 articles. Both queries were subjected to the same screening method as in PubMed, and one article was selected. Search II yielded 26 articles, none of which met the selection criteria.

In Search I of the ACM Digital Library database, the first Boolean search query ("Dementia" AND "Mobile Applications") yielded 1,634 articles, and the second Boolean search query ("Dementia" AND 
Search I

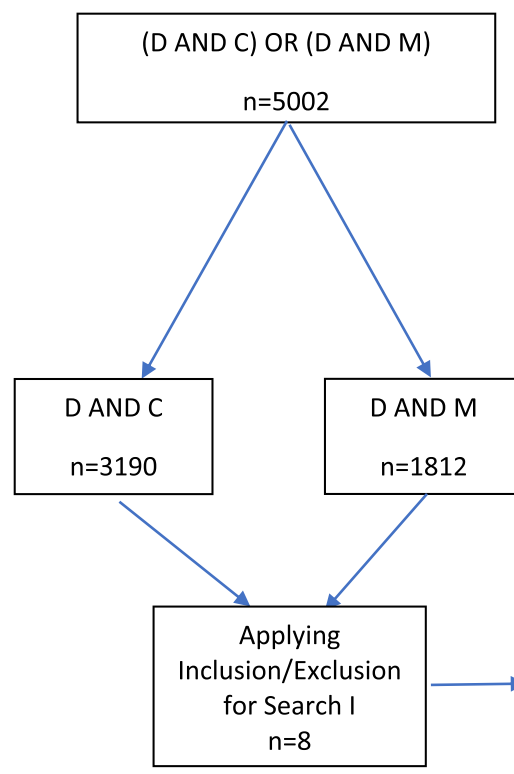

Search II

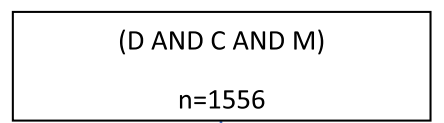

Key: (1) D = "Dementia", (2) C = "Communication", (3) M = "Mobile Applications"

Fig. 1. Search methodology and screening process.

"Communication Technology") yielded 1,576 articles, for a total of 3,210 articles. Both search queries were subjected to the same screening method as PubMed and IEEE XPLORE, and one article was selected. Search II yielded 1,498 articles, none of which met the selection criteria.

In sum, 5,002 articles were screened through Search I, and 1,556 articles were screened through Search II. After screening, there were 11 articles eligible for review, nine from PubMed, one from IEEE, and one from ACM. Duplicate articles were removed during the screening process.

Figure 1 provides a visual representation of the search methodology and screening process, combining the results from all three databases.

\section{Search terms}

In this review, the term "mobile health applications" was used in place of the more general term "mobile health technology," often truncated to the term "mobile health." Due to the novelty of this field, mobile health technology, as a term, does not have a singular definition. For the purposes of this review, the definition offered by Kumar et al. (2013) of mobile health technology as "mobile and wearable health information and sensing technologies" is sufficient
[13]. Mobile health technology is not limited to a particular set of devices and generally includes the smartphone, tablet, and wearables. Since this review focuses on communication, however, devices that are used mainly for their sensing technologies are not the primary focus and were hence discarded unless in combination with communication technologies. Such devices would include, for example, the majority of wearables to track patient movement, sleep patterns, or vital signs. While sensing technologies are increasingly being incorporated into smartphones and tablets, their use as communication devices is more common than other more sensorbased technology. Accordingly, the term "mobile health applications," or "mobile applications," as used in this search, narrows the broader range of devices to those more specific to communication.

As for the terms "dementia" and "communication technology," these two terms are relatively easy to justify. Dementia refers to the general set of symptoms in which cognitive function is impaired and/or rapidly deteriorates. It is also the primary focus of this review. Communication technology was used in the search "Dementia" AND "Communication Technology" because we wanted to encompass all types of communication technology, which would offer a broader 
understanding of its current state of integration into care arrangements. It was also used in place of the term "communication applications," because "communication applications" often refer specifically to smartphones and tablets. However, this task is already fulfilled by the term "mobile applications," making the term "communication applications" redundant in the search "Dementia" AND "Mobile Applications" AND "Communication Technology".

\section{RESULTS}

\section{Availability/efficacy/feasibility of mobile devices/applications and communication technologies in the context of dementia}

In order to assess the overall effectiveness and potential of communications-based mobile applications in supporting PwD, it is imperative to assess the current availability of mobile applications and communication technologies for this population, alongside their present feasibility and efficacy. Eight out of eleven papers are pertinent to this discussion [9, 15-21]. Two articles are systematic literature reviews that discuss currently available solutions to dementia care through mobile applications $[9,15]$. Another five of these papers specifically discuss the efficacy and feasibility of mobile applications and communication technologies related to dementia care [16-20]. The final paper is a single case study of a patient learning to interact and become autonomous with a specific mobile application [21].

\section{Availability}

With regards to the availability of mHealth applications related to the care of PwD, Maresova et al. (2018) performed a literature review that included 17 studies about different technological solutions for patients with $\mathrm{AD}$. Among those 17 papers, only one contained information about mobile devices as the type of technology used. Among these papers, the most frequent type of technology are wearable devices, such as smartwatches with embedded sensors to track the patients' location, vital signs, diet and sleep patterns, among others [9]. The one paper that did discuss the use of mobile devices concerned Ambient Assisted Living technologies (AAL) that utilized mobile devices for similar sensing and tracking purposes such as GPS [14]. The authors finalize with a discussion about the benefits and limitations of technological solutions for patients with $\mathrm{AD}$, which can also be extrapolated to feasibility of mobile devices/applications. Some of the benefits include enhancing the physical and mental wellbeing of patients and caregivers, facilitating patients' autonomy therein prolonging their independent living at home and reducing the burden on the healthcare systems. The main limitations are the difficulty of privacy preservation inherent to these technologies, the potential of PwD rejecting or being reluctant to learn how to use the application, and the current lack of strong clinical evidence.

Martínez-Alcalá et al. (2016) systematically reviewed the literature regarding ICT and the opportunities it offers to PwD and their families. Twenty-six studies were included in this review. The main finding presented in this paper is that out of the 14 papers that were oriented towards care for primary caregivers of patients, only three utilized mHealth applications, while the majority (12) utilized teleassistance applications. Furthermore, out of the 16 papers that were oriented towards care for patients with $\mathrm{AD}$, only one utilized mHealth applications [15], which evidences that the smartphones are currently underutilized as a support tool for PwD.

\section{Efficacy/feasibility}

Five papers discuss the efficacy of mobile applications for PwD support and a sixth one also refers to efficacy but is unique in that it is a single case study. In the first paper, Yousaf et al., examined 29 papers in the literature related to mHealth dementia applications. Six distinct sub-groups were created based on the type of care discussed in each paper, which is as follows: 1 ) activities of daily living (ADL) based cognitive training, 2) cognitive monitoring, 3) dementia screening, 4) reminiscence and socialization, 5) tracking, and 6) caregiver support [16]. General advantages of these range of applications included good usability, interactive features, privacy and confidentiality, online/offline features, cost-free availability, and multi-language support. Lack of these specific attributes, as well as general lack of instruction or information crucial to the application's efficacy, were casted as disadvantages. More broadly, the authors pointed out the scarcity of mHealth applications specific to dementia, despite the overall growth of the mHealth application market. Nevertheless, the results of the specific papers reviewed and their corresponding mobile health applications were generally positive for both patients and their caregivers.

Thorpe et al. (2016) took a similar approach, but they examined the efficacy of user-centered design 
(UCD) methods of smartphones and smartwatches in an experimental context [17]. In order to do this, they specified a set of six support features, namely, 1) Scheduling, 2) Navigation, 3) Communication, 4) Orientation, 5) Emergency help, and 6) Monitoring. These were tested among five patients and their caregivers, and data was gathered using a variety of techniques (i.e., video recordings, interaction logs, system usability scale questionnaires, logbooks, application usage logs and interviews). Efficacy metrics included perceived subjective usability, actual usability, key usability issues, and user acceptance. Patients preferred using the technology in ways they were familiar with, like buttons as opposed to swiping, and personalization of features proved useful for making the experience less overwhelming. The authors concluded that some of the advantages of mobile applications include 1) less stigmatization towards patients from others, 2) increasing familiarity with technology (with a view to a future that is increasingly pervaded by it), 3) personalization capabilities, and 4) more resistance to antiquity in the future, thereby making mobile applications functional for years to come. With regards to the patients' ability to use the range of support features, scheduling, communication and orientation features were deemed usable, whereas the navigation and emergency help features were "clearly not usable at all," at least for the devices used in their experiment.

Yamagata et al. (2013) took a student-led approach to developing dementia-related mHealth applications to explore the logistics of mHealth applications with a qualitative methodology, instead [18]. In the study, software engineering students produced a variety of mobile apps that focused on improving quality of life in PwD, generally through activities for neurological stimulation, such as cognitive tests and sharing photos and videos with caregivers and loved ones. The most frequent issue seemed to be the fact that patients tended to use their fingernails when trying to interact with the tablets' touch interfaces. A minor issue arose when patients showed difficulty discerning the images present within the application due to the size and the color of the image. These issues were resolved by trial and error and through reiterated patient exposure to the technology. As for the advantages, the paper mentioned how patients learned better in groups, and patients were more responsive when the tablet presented nostalgic material like their favorite songs or pictures of their family.

Differently, Koumakis et al. (2019) focused on the caregiving framework [19]. While their work encompassed assistive technologies in general, several conclusions were drawn specifically about mobile health and internet-based interventions. Primarily, they found that caregivers positively accepted internet and other mHealth-based interventions. In relation to feasibility, the authors also analyzed the cost-effectiveness of these dementia care frameworks and highlighted that more comprehensive and longitudinal studies are needed in order to move the field forward.

In the fifth paper, Hwang et al. (2020) studied the integration of an ICT product into care practices and assessed the existing technological frameworks of four families [20]. This ICT product was implemented through the use of a simplified tablet computer and contained the following features: 1) Call requests, 2) Events, 3) Medication alerts, 4) Messaging, 5) Photos and Videos, 6) Exercise videos, 7) Web Links, and 8) Video Calling. The study placed emphasis on the concept of appropriation through mutual adaptation, where not only did the behavior of the patient change through their interaction with the proposed technology, but such technology also changed to fit the needs of the patient. The study also analyzed "bricolage," as the method through which existing technology is modified to compensate for new technological arrangements. The authors examined these two concepts through the lens of the patient-caregiver relationship and evaluated this relationship primarily through questionnaires administered before and after the use of the ICT. Ultimately, they found that the appropriation was driven or impeded by 1) motivating individual and relational meanings, 2) learnability and resourcefulness, 3) responsive and cooperative care practices, and 4) empathy and shared power in care relationships.

In the sixth paper, discussing the efficacy of mobile applications, a single-case study regarding the ability of a person with $\mathrm{AD}$ to use a calendar application on a tablet computer was performed on a 65-year-old woman with $\mathrm{AD}$ [21]. The woman had used a paper diary to record all her daily activities, but at some point, she could not find room to write in her diary anymore. As a result, she had begun to lose crucial information necessary for her professional activities. She reported not having sufficient experience with smartphones or tablets and was initially reluctant to use a calendar application to help her organize her professional activities. The authors used three steps to facilitate the learning process: 1) Acquisition, 2) Application, and 3) Adaptation. At the end of the program (spanning 12 months and 23 appointments), 
the woman's average success rate for the use of the calendar application was $97.6 \%$, which represented an overall significant increase from $64 \%$ at her first session.

\section{Feasibility/efficacy of communications- based mobile applications in the context of dementia}

Having assessed the current availability, efficacy, and feasibility of mHealth applications for PwD support, this section further narrows the scope to evaluate mHealth applications specifically designed to facilitate communication and social engagement in PwD. Three out of eleven papers are relevant for this evaluation [22-24]. The first of these papers was a single-case study that focused on a 52-year-old patient with $\mathrm{AD}$. The study recorded conversations between the patient and her husband both through an application called GoTalk NOW, which was installed on the patient's tablet, and compared the conversational quality with and without the aid of the tablet. The app was designed to display certain images and videos as a means to stimulate conversation. With the assistance of GoTalk NOW, conversations lasted on average 13 minutes and 40 seconds longer than conversations without the app's assistance. However, the study also found that communicative initiatives (i.e., starting a new topic, making topic choices or asking relevant questions), were not affected by the mobile application (or lack thereof). Despite gaining familiarity and knowledge on use of the tablet, the study concluded that the woman was still heavily reliant on her husband to operate the device [22].

A second paper by Samuelsson et al. (2019) took a similar approach as the paper aforementioned [23]. The study analyzed three dialogues between patients and their caregivers and examined the differences in conversational quality with and without the assistance of two applications, namely, Computer Interactive Reminiscence and Communication Aid (CIRCA) and Computer Interactive Reminiscence and Communication University of Sheffield (CIRCUS), installed on tablet computers. Much like the previous study, the applications are designed to stimulate conversation using specific pictures, videos, and music files. Participants were active and intrigued and wanted to interact with the tablet. They were also more conversationally active when assisted by CIRCUS and CIRCA than without them. In addition to helping the patients, the caregivers also reported to experience less difficulties when trying to hold the conversation, and they generally found the aided conversations more enjoyable.

In the third paper, Wilson et al. (2020) explored the logistical implications of 27 mobile communication applications (cApps) in the Candian (English) iOS marketplace via 1) their ability to support personcentered communication in long-term residential care and 2) their market stability [24]. The principal findings were that 1) almost half of the augmentative and alternative communication (AAC) mobile applications evaluated in 2015 and 2017 contained 50 $74 \%$ of features that would support communication in long-term residential care, which is acceptable for a field still in its infancy, and 2) $74 \%$ of the mobile applications evaluated demonstrated market stability over a two-year period. None of the applications supported person-centered communication, and the tools utilized in the applications were not tailored to the individual user (e.g., generic photos, uniform speech dialect/accent, and so on). Furthermore, mobile applications were fairly stable in the marketplace, as over the 2-year period (2015 - 2017), "only one of the top recommended cApps from the 2015 review was replaced with a newly evaluated translation app."

\section{Table summary}

Below are two tables that summarize the results of this review. Table 1 compiles the results of the review papers discussed, while Table 2 compiles the results of the clinical studies discussed using the PICO format [25]. Wilson et al. (2020) [23] is not included in either table because it is neither a review paper nor a clinical study and is not readily compatible with the PICO format. However, it serves as a useful resource on the logistics of communications-based mobile applications, and thus, remains in the discussion.

\section{DISCUSSION}

With regards to mobile applications as aids for dementia care, the literature suggests that mobile applications are extremely versatile and not only allow patients to connect with their family, provide a basis for conversation, and improve their cognitive function, but also enable helpful tools such as facial pain detection and translation, diagnostic tools related to gait and posture, and video monitoring for fall detection $[11,16]$. The large scope of mobile applications as tools for improving care for PwD, therefore, increases the possibilities of technology embedding and allows for different areas of care to be combined and bolstered. Mobile applications also 
Table 1

Review Papers

\begin{tabular}{|c|c|c|c|}
\hline Studies & $\begin{array}{l}\text { Number of } \\
\text { Articles } \\
\text { Reviewed }\end{array}$ & Aim & Main Findings (Pertinent to this review) \\
\hline $\begin{array}{l}\text { Maresova et al., } 2018 \\
\text { [9] }\end{array}$ & 17 & $\begin{array}{l}\text { - To explore technological solutions for } \\
\text { patients with AD }\end{array}$ & $\begin{array}{l}\text { - Increased patient autonomy } \\
\text { - Lack of clinical evidence }\end{array}$ \\
\hline $\begin{array}{l}\text { Martínez-Alcalá et al., } \\
2016 \text { [15] }\end{array}$ & 26 & $\begin{array}{l}\text { - To discuss the opportunities that ICT offers } \\
\text { to PwD and their families }\end{array}$ & $\begin{array}{l}\text { - Studies discussing mHealth applications } \\
\text { were under-represented }\end{array}$ \\
\hline $\begin{array}{l}\text { Yousaf et al., } 2020 \\
\text { [16] }\end{array}$ & 29 & $\begin{array}{l}\text { - To examine the advantages and } \\
\text { disadvantages of mobile health dementia } \\
\text { applications }\end{array}$ & $\begin{array}{l}\text { - Ease of use } \\
\text { - Cost-free availability } \\
\text { - Multi-language support } \\
\text { - Scarceness of mobile health dementia } \\
\text { applications }\end{array}$ \\
\hline $\begin{array}{l}\text { Koumakis et al., } 2019 \\
\text { [19] }\end{array}$ & N/A & $\begin{array}{l}\text { - To analyze existing models of integrated } \\
\text { care for the management of PwD } \\
\text { - To explore assistive technologies related to } \\
\text { dementia care } \\
\text { - To describe the cost effectiveness of models } \\
\text { of integrated care for management of PwD }\end{array}$ & $\begin{array}{l}\text { - Internet and other mHealth- based } \\
\text { interventions positively accepted by } \\
\text { caregivers } \\
\text { - Scarcity with regards to high-quality } \\
\text { studies that discuss cost-effectiveness of } \\
\text { internet-based interventions }\end{array}$ \\
\hline
\end{tabular}

Table 2

Clinical Studies (PICO). Problem: Can the use of mobile applications improve daily function and/or communication in PWD?

\begin{tabular}{|c|c|c|c|c|}
\hline Studies & Population & Intervention & Comparison & Outcome \\
\hline $\begin{array}{l}\text { Thorpe, et al., } \\
2016 \text { [17] }\end{array}$ & $\begin{array}{l}\text { - Five patients and their } \\
\text { caregivers }\end{array}$ & $\begin{array}{l}\text { - User-centered design } \\
\text { (UCD) methods }\end{array}$ & - N/A & $\begin{array}{l}\text { - Usability of features } \\
\text { - Usefulness } \\
\text { - User acceptance }\end{array}$ \\
\hline $\begin{array}{l}\text { Yamagata et al., } \\
2013 \text { [18] }\end{array}$ & $\begin{array}{l}\text { - Older adults with AD } \\
\text { and dementia }\end{array}$ & $\begin{array}{l}\text { - Mobile applications } \\
\text { developed by students }\end{array}$ & $\bullet$ N/A & $\bullet$ N/A \\
\hline $\begin{array}{l}\text { Hwang et al., } \\
2020 \text { [20] }\end{array}$ & $\begin{array}{l}\text { - } 4 \text { patients and their } \\
\text { caregivers }\end{array}$ & $\begin{array}{l}\text { - ICT product developed by } \\
\text { small technology company }\end{array}$ & $\begin{array}{l}\text { - Before use of ICT } \\
\text { intervention }\end{array}$ & $\begin{array}{l}\text { - Metrics of Collaborative } \\
\text { Appropriation: } \\
\text { 1. Motivated meanings } \\
\text { 2. Learnability of } \\
\text { technology } \\
\text { 3. Establishment of } \\
\text { responsive and } \\
\text { cooperative care } \\
\text { practices } \\
\text { 4. Qualities of empathy and } \\
\text { shared power in } \\
\text { relationships }\end{array}$ \\
\hline $\begin{array}{l}\text { Imbeault et al., } \\
2018[21]\end{array}$ & $\begin{array}{l}\text { - } 65 \text {-year- old woman } \\
\text { with AD }\end{array}$ & $\begin{array}{l}\text { - Use of calendar application } \\
\text { over 12-month period }\end{array}$ & $\begin{array}{l}\text { Diagnostic test before } \\
\text { learning how to use the } \\
\text { calendar application }\end{array}$ & $\begin{array}{l}\text { - Success rate for use of } \\
\text { calendar application } \\
\text { - Ability to retain information }\end{array}$ \\
\hline $\begin{array}{l}\text { Ekström et al., } \\
2017 \text { [22] }\end{array}$ & $\begin{array}{l}\text { - 52-year- old patient } \\
\text { with AD }\end{array}$ & $\begin{array}{l}\text { - Aid of application GoTalk } \\
\text { NOW }\end{array}$ & $\begin{array}{l}\text { - Without the aid of } \\
\text { application GoTalk NOW }\end{array}$ & $\begin{array}{l}\text { - Quality of conversation } \\
\text { - Communicative initiatives } \\
\text { - Length of conversation }\end{array}$ \\
\hline $\begin{array}{l}\text { Samuelsson } \\
\text { et al., } 2019 \\
\text { [23] }\end{array}$ & $\begin{array}{l}\text { - Three dyads consisting } \\
\text { of patient and caregiver }\end{array}$ & $\begin{array}{l}\text { - Assistance of Computer } \\
\text { Interactive Reminiscence } \\
\text { and Communication Aid } \\
\text { (CIRCA) } \\
\text { - Assistance of Computer } \\
\text { Interactive Reminiscence } \\
\text { and Communication } \\
\text { University of Sheffield } \\
\text { (CIRCUS) }\end{array}$ & $\begin{array}{l}\text { - Without Assistance of } \\
\text { Computer Interactive } \\
\text { Reminiscence and } \\
\text { Communication Aid } \\
\text { (CIRCA) } \\
\text { - Without Assistance of } \\
\text { Computer Interactive } \\
\text { Reminiscence and } \\
\text { Communication University } \\
\text { of Sheffield (CIRCUS) }\end{array}$ & - Quality of conversation \\
\hline
\end{tabular}


prove to be relatively feasible and logistically convenient, as they require little infrastructure and can be used ubiquitously. Furthermore, applications can be relatively inexpensive or even free of cost, easy to use, free of social stigma among patients, and stable in the marketplace $[16,17,24]$.

The literature also indicates that there is a growing marketplace for mHealth applications, which could host new and improved applications related to dementia care [16], since their current lack of availability seems to be the major downfall of the field $[9,15]$. From an app development perspective, the evidence suggests that cApps have considerable room for improvement. For instance, dialogue systems and automatic speech recognition technologies are still not optimized for dementia speech, which often contains mistakes and agrammatical sentences [26]. Besides, many of the features that constitute true person-centered communication (e.g., text-to-speech functionality, two-way communication, personalized text, or videos) are missing and need to be integrated for the apps to become relevant and helpful to their target users [24]. Well-designed interfaces and enhanced usability are imperative in application development, as PwD are not only afflicted by severe learning difficulties associated with the disease, but also tend to be relatively unfamiliar with such technology, given the increased prevalence of dementia amongst older populations. The literature also shows the need for mobile application developers to adapt to patient needs, especially regarding application layout and comprehension concerns [18].

The reviewed papers ultimately suggest that the efficacy of communications-based mobile applications and patient autonomy depends, in large part, on the individual needs of each patient. Factors such as stage of dementia, prior experience with technology, and social acceptability all play a role in whether the patient can become autonomous with the mobile application and improve their communication abilities. Despite there being an added burden to the caregiver of operating and using the mobile application, it seems to pale in comparison to the reduction in burden and increase in happiness in caregivers who have had success using these applications. It must be noted that patient autonomy in use of communications-based mobile applications intended for emergency use or for expressing their needs or desires may be a primary goal, especially in non-verbal patients and/or if intended for emergency use or for expressing their needs or desires. Once again, the efficacy of these applications relies heavily on the capability and circumstance of the individual patient. Exposure, repetition, and practice with the technology can yield positive results in PwD. This includes increased liveliness when using the applications, more activity during conversation, and more familiarity and overall proficiency with the technology $[18,21,22]$. Given the apathy, dysphoria, and depression associated with dementia and the association of these difficulties with reduced social engagement and loneliness, communications-based mobile applications are a promising mitigation strategy [27]. Ultimately, if patient autonomy is achieved, it would reduce emotional burden on patients and caregivers.

The literature also highlights the role of the caregiver for technological interventions to be successful. The integration of these interventions relies not only on the patient's comfort, acceptability, and familiarity, but also on the caregiver. As has been shown, caregivers have positively accepted internet and mobile-based interventions [19], which is also promising for more complex communications-based interventions. Additionally, the dynamic caregiverpatient relationship has been shown to change in order to accommodate technological interventions [20]. As patients and caregivers become comfortable with new technologies, they discover new ways to spend time with each other (and improve quality of time), communicate their difficulties openly, and learn and grow constructively, thereby improving overall care and life quality. Ultimately, the ways in which patients and caregivers benefit from technology are intertwined, as they depend on each other to make progress both individually and relationally.

It is important to highlight some limitations with this review and justify the methodology. This review is not systematic, partially due to the youth and heterogeneity of the field, as well as to the scarcity of available publications. Only three databases were considered, namely, PubMed, IEEE XPLORE, and ACM Digital Library, aiming for the intersection between clinical and technical journals. In addition, as implied earlier, the lack of papers discussed in this review that specifically relate to communicationsbased mobile applications reflects the fact that any claims made in this paper are not generalizable, especially those concerning the availability of mobile applications, due to the sparse set of literature selected and the combined limitations of the methodologies of the papers reviewed.

The original aim of this paper was to assess the efficacy of smartphone applications as communication 
aids for people with dementia. As a means to increase the number of articles included, the search was expanded to separately include smartphone applications aids for dementia care and communication technology as aids for dementia care. For example, in the PubMed search, just using Search II would only have amassed to a pool of 32 articles to be screened. Just by using a different Boolean search query, Search I yielded 1,510 more articles that could be screened. In addition, despite using the same search terms, the two searches explored vastly different content. Search I sought to lay a foundation for the current dementia care techniques implemented through the mediums of mobile applications and communication technology, respectively, and how available and feasible those mediums were within the market. Search II narrowed the approach to how available and feasible communications-based mobile applications were within the market and the conclusions drawn from these articles could be conglomerated with the conclusions drawn from the articles in Search I. For example, articles part of Search I, such as the paper by Imbeault et al. (2018), that discussed a patient's interaction with a non-communication based mobile application could be extrapolated to a patient's interaction with a communications-based mobile application because the delivery of the care technique is through the same medium [21]. In total, creating two searches allowed for a progression from a broader view of mHealth applications and the status of cApps within that market to a more focused view of cApps in social and clinical settings. Accordingly, this paper was structured to accommodate for such progressions of scope.

It is also important to note that citation searching was explored in the preliminary stages of this review. However, there were two main reasons why it was not considered in the final search. Firstly, when searching citations from larger reviews, most of the articles discussed singular mobile applications that, either did not concern communication or were not applied in a clinical setting or both. Secondly, citation searching original papers resulted in articles that were not particularly relevant to this review. This was because the very original literature search only searched for articles that fulfilled the search criterion "Dementia" AND "Mobile Applications" AND "Communication Technology." With such a specific- search criterion, along with the relative novelty and heterogeneity of this field, most if not all articles did not meet the selection criteria.
Despite these changes, much of the literature was rejected, and this was due to a couple of factors at play. First, the lack of development of communicationsbased mobile applications. And second, most of the literature lacked human involvement (patient or caregiver) with the mobile technology. There were numerous articles about individual mobile applications and their features, but they had not been applied in the clinical setting, which speaks to the current youth of the field and the need for studies aimed at bridging the gap between technical research and clinical contexts. These articles were rejected because they did not fit in with the role of this paper, which was to document both the logistics and feasibility of mobile applications and the clinical results of communications-based mobile applications, which would yield the best measures of efficacy. Therefore, the lack of patient or caregiver involvement was the greatest limiting factor in the literature search and led to the greatest number of rejections. It is also crucial to note that several articles included in this review did not incorporate human involvement into the assessment of mobile applications, but this was because these types of articles were larger reviews that offered a broader perspective on the feasibility and/or availability of communications-based mobile applications, as a whole $[9,15,16]$.

Given the results obtained from this review, one conclusion is evident: the need for further research and communications-based mobile application development for people with dementia, especially translational research. Compared to the rest of applications available in the mobile-health market, applications for dementia care (specifically communications-related dementia care) are concerningly sparse, despite their potential to mitigate the detrimental impact of deteriorating social interactions on the wellbeing of PwD and their caregivers. Some of the mentioned advantages of this technology include increased liveliness in patients, cost-effectiveness, ease of use, general facilitation of conversation, and enhancement of caregiver-patient relationships. However, it has been shown that there is still considerable room for improvement among app developers in terms of features designed for user specialization and person-centered communication. These lackluster outcomes raise further questions as to whether communications-based mobile applications are more difficult to develop than other mHealth apps or whether it is not a primary area of interest. The problem becomes exacerbated by the lack of clin- 
ical data in the feasibility studies of these apps, as well as the scarce dialogue between app developers and communication experts, clinicians, and the target population. One obstacle could include the lack of availability from caregivers and patients to partake in these kinds of studies due to stigma, disruption of current care arrangements, time burden, incompatibility with other responsibilities or reluctance to learn and incorporate novel technology. Without feedback from clinicians, patients, their families, and their caregivers, mobile app developers cannot assess how to improve their technology or upscale it to a larger dementia population. Therefore, the availability of communications-based mobile applications relies on a careful interplay between the app developer, clinical researchers, caregivers and patients' families, and most importantly, the patients themselves. It is difficult to pinpoint exactly where this interplay fails and why cApps are so scarce within the broader mHealth application market and literature. Consequently, we suggest that a future research avenue should comprehensively address this interplay.

The most important input comes from the patient and the caregiver. Accordingly, it would be useful to understand what types of care arrangements caregivers currently employ, what they would like to improve upon and what types of interventions do they and their patients prefer. This information can be gleaned from surveys, and it would provide app developers and clinicians an idea of any unmet needs that caregivers and patients perceive in their care arrangements and how best to implement and structure interventions that target those areas in a manner that suits individual caregiver-patient groups. A possible outcome could reveal that, perhaps, patients and caregivers do not prefer technological interventions or feel that communication is not as much of a priority as ambient assisted living technologies or other tracking technologies. However, as far as this review has shown, caregivers are more than willing to accept technological interventions, and the improvement in patients' communication abilities certainly reduces burden in caregivers. If anything, this kind of survey will provide transparency in terms of caregiver and patient needs.

There is intent on the part of mobile health app developers, clinicians, and patients and caregivers to develop, document, and improve communication in patients. However, the failure lies in the execution and feasibility. As has been shown in this review, there is a very steep learning curve associated with technology and mobile-based interventions in dementia patient populations. Furthermore, while progress has certainly been made, tailoring mobile applications to the patient's needs remains a very challenging objective. Communications-based mobile applications add yet another difficulty because they require patient interaction unlike sensor-based technology. To further complicate matters, some communications-based mobile applications must reliably analyze speech patterns from various patients with vastly different speech pathologies. A feasible first step would be to increase research findings related to verbal speech patterns and non-verbal behavior patterns in PwD with a view to design better frameworks for cApps [28]. The goal, ultimately, is to improve quality of life in patients and their caregivers, of which a significant aspect involves enhancing their communication abilities, thereby enhancing their social interactions.

\section{CONFLICT OF INTEREST}

The authors have no conflict of interest to report.

\section{REFERENCES}

[1] (2020) 2020 Alzheimer's disease facts and figures. Alzheimers Dement 16, 391-460.

[2] Tisher A, Salardini A (2019) A comprehensive update on treatment of dementia. Semin Neurol 39, 167-178.

[3] Liu S, Li C, Shi Z, Wang X, Zhou Y, Liu S, Liu J, Yu T, Ji Y (2017) Caregiver burden and prevalence of depression, anxiety and sleep disturbances in Alzheimer's disease caregivers in China. J Clin Nurs 26, 1291-1300.

[4] Mahendra N, Engineer N (2009) Effects of vascular dementia on cognition and linguistic communication: A case study. Perspect Neurophysiol Neurogenic Speech Lang Disord 19, 107-116.

[5] Kirshner HS (2012) Primary progressive aphasia and Alzheimer's disease: Brief history, recent evidence. Curr Neurol Neurosci Rep 12, 709-714.

[6] Rousseaux M, Sève A, Vallet M, Pasquier F, MackowiakCordoliani MA (2010) An analysis of communication in conversation in patients with dementia. Neuropsychologia 48, 3884-3890.

[7] Lindberg B, Nilsson C, Zotterman D, Söderberg S, Skär L (2013) Using information and communication technology in home care for communication between patients, family members, and healthcare professionals: A systematic review. Int J Telemed Appl 2013, 461829.

[8] Lucero RJ, Fehlberg EA, Patel AGM, Bjarnardottir RI, Williams R, Lee K, Ansell M, Bakken S, Luchsinger JA, Mittelman M (2019) The effects of information and communication technologies on informal caregivers of persons living with dementia: A systematic review. Alzheimers Dement (N Y) 5, 1-12. 
[9] Maresova P, Tomsone S, Lameski P, Madureira J, Mendes A, Zdravevski E, Chorbev I, Trajkovik V, Ellen M, Rodile K (2018) Technological solutions for older people with Alzheimer's disease: Review. Curr Alzheimer Res 15, 975-983.

[10] Cunningham S, Brill M, Whalley JH, Read R, Anderson G, Edwards S, Picking R (2019) Assessing wellbeing in people living with dementia using reminiscence music with a mobile app (Memory Tracks): A mixed methods cohort study. J Healthc Eng 2019, 8924273.

[11] Serra-Añó P, Pedrero-Sánchez JF, Hurtado-Abellán J, Inglés M, Espí-López GV, López-Pascual J (2019) Mobility assessment in people with Alzheimer disease using smartphone sensors. J Neuroeng Rehabil 16, 103.

[12] Klimova B (2017) Mobile phone apps in the management and assessment of mild cognitive impairment and/or mildto-moderate dementia: An opinion article on recent findings. Front Hum Neurosci 11, 461.

[13] Kumar S, Nilsen WJ, Abernethy A, Atienza A, Patrick K, Pavel M, Riley WT, Shar A, Spring B, Spruijt-Metz D, Hedeker D, Honavar V, Kravitz R, Craig Lefebvre R, Mohr DC, Murphy SA, Quinn C, Shusterman V, Swendeman D (2013) Mobile Health Technology Evaluation: The mHealth evidence workshop. Am J Prev Med 45, 228-236.

[14] Blackman S, Matlo C, Bobrovitskiy C, Waldoch A, Fang ML, Jackson P, Mihailidis A, Nygård L, Astell A, Sixsmith A (2016) Ambient assisted living technologies for aging well: A scoping review. J Intell Syst 25, 55-69.

[15] Martínez-Alcalá CI, Pliego-Pastrana P, Rosales-Lagarde A, Lopez-Noguerola J, Molina-Trinidad EM (2016) Information and communication technologies in the care of the elderly: Systematic review of applications aimed at patients with dementia and caregivers. JMIR Rehabil Assist Technol 3, e6.

[16] Yousaf K, Mehmood Z, Awan IA, Saba T, Alharbey R, Qadah T, Alrige MA (2020) A comprehensive study of mobile-health based assistive technology for the healthcare of dementia and Alzheimer's disease (AD). Health Care Manag Sci 23, 287-309.

[17] Thorpe JR, Rønn-Andersen KVH, Bień P, Özkil AG, Forchhammer BH, Maier AM (2016) Pervasive assistive technology for people with dementia: A UCD case. Healthc Technol Lett 3, 297-302.
[18] Yamagata C, Coppola JF, Kowtko M, Joyce S (2013) Mobile app development and usability research to help dementia and Alzheimer patients. 2013 IEEE Long Island Systems, Applications and Technology Conference (LISAT), pp. 1-6.

[19] Koumakis L, Chatzaki C, Kazantzaki E, Maniadi E, Tsiknakis M (2019) Dementia care frameworks and assistive technologies for their implementation: A review. IEEE Rev Biomed Eng 12, 4-18.

[20] Hwang AS, Jackson P, Sixsmith A, Nygård L, Astell A, Truong KN, Mihailidis A (2020) Exploring how persons with dementia and care partners collaboratively appropriate information and communication technologies. ACM Trans Comput-Hum Interact 27, 1-38.

[21] Imbeault H, Langlois F, Bocti C, Gagnon L, Bier N (2018) Can people with Alzheimer's disease improve their dayto-day functioning with a tablet computer? Neuropsychol Rehabil 28, 779-796.

[22] Ekström A, Ferm U, Samuelsson C (2017) Digital communication support and Alzheimer's disease. Dementia 16, 711-731.

[23] Samuelsson C, Ekström A (2019) Digital communication support in interaction involving people with dementia. Logop Phoniatr Vocology 44, 41-50.

[24] Wilson R, Cochrane D, Mihailidis A, Small J (2020) Mobile apps to support caregiver-resident communication in longterm care: Systematic search and content analysis. JMIR Aging 3, e17136.

[25] Amir-Behghadami M, Janati A (2020) Population, Intervention, Comparison, Outcomes and Study (PICOS) design as a framework to formulate eligibility criteria in systematic reviews. Emerg Med J 37, 387-387.

[26] Xiong W, Droppo J, Huang X, Seide F, Seltzer M, Stolcke A, Yu D, Zweig G (2016) Achieving human parity in conversational speech recognition. arXiv, 1610.05256

[27] Landes AM (2005) Prevalence of apathy, dysphoria, and depression in relation to dementia severity in Alzheimer's disease. J Neuropsychiatr 17, 342-349.

[28] de la Fuente Garcia S, Ritchie C, Luz S (2020) Artificial intelligence, speech, and language processing approaches to monitoring Alzheimer's disease: A systematic review. $J$ Alzheimers Dis 78, 1547-1574. 